Supporting Information:

\title{
A Computational study of urea and its homologue glycinamide: Conformations, rotation barriers and relative interactions with sodium chloride
}

\section{Ajeet Singh, Shampa Chakraborty and Bishwajit Ganguly*}

Table S1: Relative energies calculated for urea conformers

\begin{tabular}{|l|l|l|l|}
\hline Method & (I) & (II) & (III) \\
\hline Imaginary freq. & 1 & 0 & 2 \\
\hline$\Delta \mathrm{E}$ & $1.0(0.0)$ & $0.0(.10)$ & $1.20(.10)$ \\
\hline$\Delta \mathrm{Ho}$ & 0.20 & 0.20 & 0.0 \\
\hline$\Delta \mathrm{H}_{298}$ & 0.50 & 0.50 & 0.0 \\
\hline$\Delta \mathrm{G}_{298}$ & 0.00 & 0.00 & 0.30 \\
\hline$\mu_{\mathrm{D}}$ & $4.50(5.60)$ & $3.80(4.80)$ & $4.50(5.70)$ \\
\hline $\mathrm{B} 3 \mathrm{LYP} / 6311++\mathrm{G}^{* *}$ & & & \\
\hline$\Delta \mathrm{E}$ & 0.80 & 0.0 & 1.20 \\
\hline $\mathrm{MP} 2 / 6-311++\mathrm{G}^{* *}$ & & & \\
\hline$\Delta \mathrm{E}$ & 1.40 & 0.0 & 2.60 \\
\hline
\end{tabular}

$\Delta \mathrm{E}=$ relative energy $(\mathrm{kcal} / \mathrm{mol}) ; \Delta \mathrm{Ho}=$ relative energy with zero-point vibration correction (kcal/mol);

$\Delta \mathrm{H}_{298}=$ relative energy corrected for thermal vibrations $(\mathrm{kcal} / \mathrm{mol})$;

$\Delta \mathrm{G}_{298}=$ relative free energy $(\mathrm{kcal} / \mathrm{mol}) ; \mu_{\mathrm{D}}=$ dipole moment (debye).

Solvent calculated results are in parentheses.

Table S2: Relative energies calculated for glycinamide conformers (IV-VII)

\begin{tabular}{|c|c|c|c|c|}
\hline Method & (IV) & (V) & (VI) & (VII) \\
\hline \multicolumn{5}{|l|}{ B3LYP/6-311+G* } \\
\hline Imaginary freq & 0 & 0 & 0 & 0 \\
\hline$\Delta \mathrm{E}$ & 0.0 & $3.70(2.10)$ & $2.30(1.70)$ & $3.40(2.80)$ \\
\hline$\Delta \mathrm{Ho}$ & 0.0 & 3.20 & 1.70 & 2.90 \\
\hline$\Delta \mathrm{H}_{298}$ & 0.0 & 3.40 & 2.10 & 3.10 \\
\hline$\Delta \mathrm{G}_{298}$ & 0.0 & 3.0 & 0.80 & 2.70 \\
\hline$\mu_{\mathrm{D}}$ & $4.10(4.90)$ & $4.20(5.40)$ & $3.40(4.30)$ & $3.70(4.70)$ \\
\hline \multicolumn{5}{|l|}{ B3LYP/6-311+G** } \\
\hline Imaginary freq & 0 & 0 & 0 & 0 \\
\hline$\Delta \mathrm{E}$ & 0.0 & 3.40 & 2.10 & 3.0 \\
\hline$\Delta \mathrm{Ho}$ & 0.0 & 2.90 & 1.60 & 2.70 \\
\hline
\end{tabular}




\begin{tabular}{|l|l|l|l|l|}
\hline$\Delta \mathrm{H}_{298}$ & 0.0 & 3.10 & 1.90 & 2.80 \\
\hline$\Delta \mathrm{G}_{298}$ & 0.0 & 2.80 & 0.80 & 2.40 \\
\hline$\mu_{\mathrm{D}}$ & 4.10 & 4.20 & 3.50 & 3.70 \\
\hline $\mathrm{B} 3 \mathrm{LYP} / 6-311++\mathrm{G}^{* *}$ & & & & \\
\hline$\Delta \mathrm{E}$ & 0.00 & 3.40 & 2.10 & 3.0 \\
\hline $\mathrm{MP} 2 / 6-311++\mathrm{G}^{* *}$ & & & & \\
\hline$\Delta \mathrm{E}$ & 0.00 & 3.80 & 2.0 & 2.80 \\
\hline
\end{tabular}

$\Delta \mathrm{E}=$ relative energy $(\mathrm{kcal} / \mathrm{mol}) ; \Delta \mathrm{Ho}=$ relative energy with zero-point vibration correction $(\mathrm{kcal} / \mathrm{mol}) ; \Delta \mathrm{H}_{298}=$ relative energy corrected for thermal vibrations $(\mathrm{kcal} / \mathrm{mol}) ; \Delta \mathrm{G}_{298}=$ relative free energy $(\mathrm{kcal} / \mathrm{mol}) ; \mu_{\mathrm{D}}=$ dipole moment (debye). Solvent calculated results are in parentheses.

Table S3: Selected geometrical parameters at B3LYP/6-311+G* and at B3LYP/6$311+\mathrm{G}^{* *}$ levels for Glycinamide conformers

\begin{tabular}{|l|l|l|l|c|}
\hline & \multicolumn{1}{|c|}{$(\mathbf{I V )}$} & $\mathbf{( V )}$ & $\mathbf{( V I )}$ & $\mathbf{( V I I )}$ \\
\hline $\mathrm{C}(2)-\mathrm{N}(1)$ & $1.467(1.467)$ & $1.453(1.454)$ & $1.450(1.450)$ & $1.456(1.456)$ \\
\hline $\mathrm{C}(2)-\mathrm{C}(7)$ & $1.533(1.534)$ & $1.538(1.538)$ & $1.540(1.539)$ & $1.528(1.528)$ \\
\hline $\mathrm{C}(7)-\mathrm{N}(9)$ & $1.356(1.357)$ & $1.363(1.363)$ & $1.360(1.366)$ & $1.363(1.364)$ \\
\hline $\mathrm{C}(7)-\mathrm{O}(8)$ & $1.220(1.220)$ & $1.219(1.219)$ & $1.218(1.219)$ & $1.218(1.218)$ \\
\hline $\mathrm{C}(7)-\mathrm{C}(2)-\mathrm{N}(1)$ & $113.5(113.7)$ & $120.8(120.6)$ & $115.4(115.3)$ & $109.2(109.7)$ \\
\hline $\mathrm{N}(9)-\mathrm{C}(7)-\mathrm{C}(2)$ & $114.5(114.7)$ & $116.7(116.7)$ & $114.9(115.0)$ & $115.6(115.5)$ \\
\hline $\mathrm{N}(9)-\mathrm{H}(10)-\mathrm{H}(11)$ & $121.1(121.6)$ & $118.9(119.4)$ & $118.2(118.7)$ & $118.3(118.7)$ \\
\hline $\mathrm{H}(11)-\mathrm{N}(9)-\mathrm{C}(7)$ & $119.5(119.2)$ & $118.7(118.6)$ & $119.0(118.8)$ & $118.5(118.3)$ \\
\hline $\mathrm{H}(10)-\mathrm{N}(9)-\mathrm{C}(7)$ & $119.2(119.0)$ & $122.2(121.9)$ & $122.5(122.3)$ & $122.4(122.0)$ \\
\hline $\mathrm{N}(1)-\mathrm{C}(2)-\mathrm{C}(7)-\mathrm{N}(9)$ & $-14.2(-14.1)$ & $0.0(0.00)$ & $-171.0(-168.0)$ & $-150.0(-152.0)$ \\
\hline $\mathrm{N}(1)-\mathrm{C}(2)-\mathrm{C}(7)-\mathrm{O}(8)$ & $166.8(116.9)$ & $180.0(180.0)$ & $8.7(12.4)$ & $32.2(30.1)$ \\
\hline $\mathrm{C}(2)-\mathrm{C}(7)-\mathrm{N}(9)-\mathrm{H}(11)$ & $-176.0(-176.0)$ & $-179.0(-179.0)$ & $-176.0(-176.0)$ & $-179.0(-179.0)$ \\
\hline $\mathrm{C}(2)-\mathrm{C}(7)-\mathrm{N}(9)-\mathrm{H}(10)$ & $-1.2(-1.0)$ & $0.0(0.0)$ & $-0.7(0.0)$ & $1.3(11.5)$ \\
\hline $\mathrm{H}(5)-\mathrm{N}(1)-\mathrm{C}(2)-\mathrm{C}(7)$ & $-88.1(-88.2)$ & $64.7(64.7)$ & $62.2(64.4)$ & $-36.4(-33.9)$ \\
\hline $\mathrm{H}(6)-\mathrm{N}(1)-\mathrm{C}(2)-\mathrm{C}(7)$ & $152.1(151.7)$ & $-64.6(64.7)$ & $-53.9(-51.7)$ & $-156.0(-153.0)$ \\
\hline $\mathrm{H}(3)-\mathrm{C}(2)-\mathrm{C}(7)-\mathrm{N}(9)$ & $105.2(105.3)$ & $124.1(124.1)$ & $-48.6(-44.9)$ & $-29.4(-31.4)$ \\
\hline $\mathrm{H}(4)-\mathrm{C}(2)-\mathrm{C}(7)-\mathrm{N}(9)$ & $-141.0(-141.0)$ & $-124.0(-124)$ & $66.2(69.8)$ & $85.7(83.6)$ \\
\hline
\end{tabular}

$\mathrm{B} 3 \mathrm{LYP} / 6-311+\mathrm{G}^{* *}$ values are in parentheses. Bond lengths in angstrom and bond angles in degrees, respectively. 
Rotational Barriers for Glycinamide Conformers: The rotational and inversion barriers for the glycinamide conformers from IV to $\mathbf{V}$ and to $\mathbf{V I}$ have been investigated at B3LYP/6-311+G** level (Figure S1). The relative transition state free energies calculated with respect to the most stable glycinamide conformer IV for the bond rotations and inversions are summarized in Figure S1. The nitrogen $\left(\mathrm{N}_{1}\right)$ inversion barrier VIII calculated is relatively smaller than that of bond rotation $\mathrm{C}_{2}-\mathrm{N}_{1}$ IX between the conformers IV and $\mathbf{V}$ (Figure S1a). Moving to the rotational barrier between the conformers IV and VI, transition states have been located at B3LYP/6-311+G** level. On the potential energy surface, two transition states have been predicted (Figure S1b). First transition state has been located for the $\mathrm{C}_{2}-\mathrm{C}_{7}$ bond rotation $\mathbf{X}$ followed by the intermediate VII, which moves to another transition state $\mathbf{X I}$ via $\mathrm{C}_{2}-\mathrm{N}_{1}$ bond rotation and eventually leads to the conformer VI (Figure S1b).

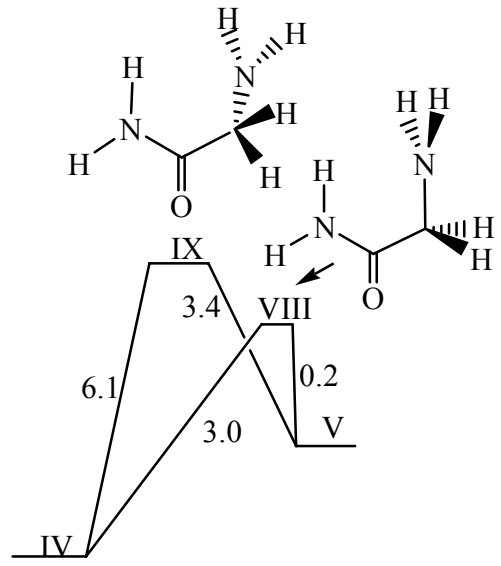

(a)

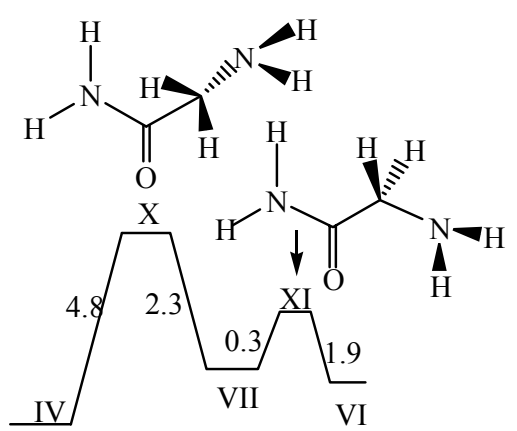

(b)

Figure S1: Calculated Free energy difference $\left(\Delta \mathrm{G}_{298}\right)$ at B3LYP/6-311+G** for the rotational isomers: (a) (IV) to (V) and (b) (IV) to (VI), respectively. 
Table S4: Relative energies calculated for the conformers calculated at different DFT levels (kcal/mol).

\begin{tabular}{lccccc}
\hline \multicolumn{1}{c}{ urea } & I & & II & III & \\
B3LYP/6-311+G* & 1.0 & & 0.0 & 1.2 & \\
LDA/PWC/DND & 1.2 & \multicolumn{2}{c}{0.0} & 1.6 & \\
glycinamide & IV & V & VI & & VII \\
B3LYP/6-311+G* & 0.0 & 3.7 & 2.3 & & 3.4 \\
LDA/PWC/DND & 0.0 & 3.8 & 2.9 & & 3.5 \\
\hline
\end{tabular}

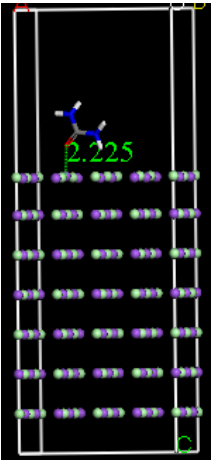

(a)

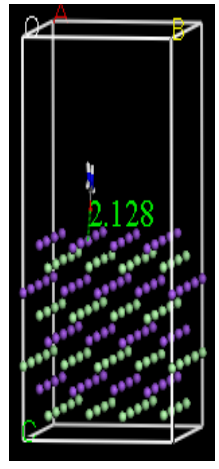

(b)

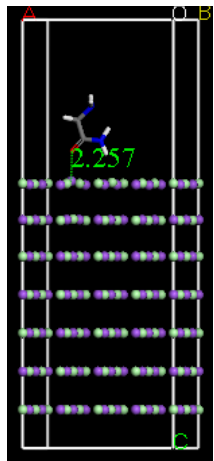

(c)

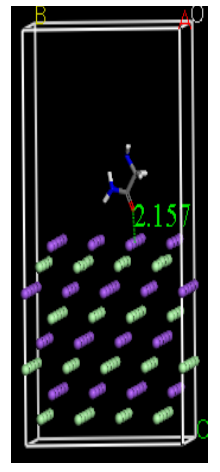

(d)

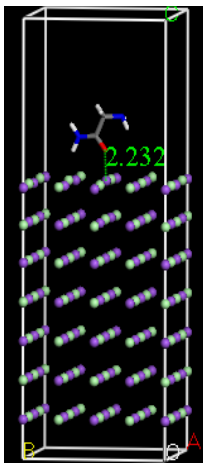

(e)

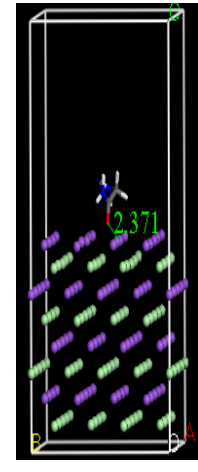

(f)

Figure S2: LDA/PWC/DND calculated geometries of urea II (a-b), glycinamide conformers IV (c-d) and VI (e-f) with (100)/(111) top two-layered relaxed surface of sodium chloride in slab models (purple sodium, green chlorine, red oxygen, blue nitrogen and white hydrogen). 


\section{Optimized geometries of urea conformers at B3LYP/6-311+G* level}

Figure 1:

Urea C2 (II)

$\begin{array}{lrrr}\mathrm{C} & 0.000000 & 0.000000 & 0.498223 \\ 0 & 0.00000 \odot & 0.000000 & 1.718718 \\ \mathrm{~N} & -0.923916 & -0.690675 & -0.251168 \\ \mathrm{H} & -0.630904 & -1.039035 & -1.167534 \\ \mathrm{H} & -1.459087 & -1.358233 & 0.310233 \\ \mathrm{~N} & 0.923916 & 0.690675 & -0.251168 \\ \mathrm{H} & 1.459087 & 1.358233 & 0.310233 \\ \mathrm{H} & 0.630904 & 1.039035 & -1.167534\end{array}$

Urea C2 (II) in water

$\begin{array}{lr}\mathrm{C} & 0.000257 \\ 0 & 0.000091 \\ \mathrm{~N} & -0.912708 \\ \mathrm{H} & -0.722945 \\ \mathrm{H} & -1.422386 \\ \mathrm{~N} & 0.913397 \\ \mathrm{H} & 1.421604 \\ \mathrm{H} & 0.722690\end{array}$

$\begin{array}{rr}-0.000191 & 0.501293 \\ -0.000059 & 1.738663 \\ -0.703955 & -0.220940 \\ -0.931818 & -1.201833 \\ -1.422210 & 0.302660 \\ 0.703628 & -0.220908 \\ 1.422954 & 0.302662 \\ 0.931650 & -1.201597\end{array}$

Urea CS (I)

$\begin{array}{rr}\mathrm{C} & -0.340907 \\ 0 & -1.282909 \\ \mathrm{~N} & 0.248037 \\ \mathrm{H} & -0.288211 \\ \mathrm{~N} & 0.248037 \\ \mathrm{H} & -0.288211 \\ \mathrm{H} & 0.852082 \\ \mathrm{H} & 0.852082\end{array}$

$-\odot .387402$

0.000000

$-1.165929$

$\odot .000000$

$\odot .051273$

1.156123

$-0.148921$

2.002003

0.051273

$-1.156123$

$-0.148921$

$-2.002003$

$\odot .874313$

$-1.173721$

$\odot .874313$

1.173721

Urea CS (I) in water

$\begin{array}{rr}\mathrm{C} & -0.333592 \\ \mathrm{O} & -1.285752 \\ \mathrm{~N} & 0.246275 \\ \mathrm{H} & -0.285730 \\ \mathrm{~N} & 0.246243 \\ \mathrm{H} & -0.285906 \\ \mathrm{H} & 0.849441 \\ \mathrm{H} & 0.849020\end{array}$

-0.381958
-1.174287
0.044044
-0.137856
0.044016
-0.137603
0.871654
0.871989

$-0.000018$

$\odot .000026$

1. 151827

2. 007607

$-1.151878$

$-2.007516$

$-1.160378$

1.160331

Urea C2v (III) 


\begin{tabular}{|c|c|c|c|}
\hline C & $\odot .0 \odot 0 \odot \odot \odot$ & $\odot .00 \odot \odot \odot \odot$ & -0.516730 \\
\hline 0 & $\odot . ๑ \odot \odot \odot \odot \odot$ & $\odot .0 \odot \odot \odot \odot \odot$ & -1.739518 \\
\hline $\mathrm{N}$ & $\odot . ๑ \odot \odot \odot \odot \odot ~$ & 1.152984 & 0.213457 \\
\hline $\mathrm{H}$ & $\odot . ๑ \odot \odot \odot \odot \odot ~$ & 2.023477 & -0.317320 \\
\hline $\mathrm{N}$ & $\odot .0 \odot \odot \odot \odot \odot$ & -1.152984 & 0.213457 \\
\hline $\mathrm{H}$ & $\odot . ๑ \odot \odot \odot \odot \odot ~$ & -2.023477 & -0.317320 \\
\hline H & 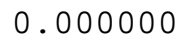 & -1.191153 & 1.231987 \\
\hline H & 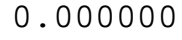 & 1.191153 & 1.231987 \\
\hline
\end{tabular}

Urea C2v (III) in water

$\begin{array}{lrrr}\mathrm{C} & -0.000006 & -0.000032 & -0.507731 \\ \mathrm{O} & -0.000006 & 0.000032 & -1.748540 \\ \mathrm{~N} & -0.000034 & 1.148412 & 0.207167 \\ \mathrm{H} & 0.000008 & 2.031072 & -0.308284 \\ \mathrm{~N} & 0.000035 & -1.148462 & 0.207171 \\ \mathrm{H} & 0.000011 & -2.031089 & -0.308297 \\ \mathrm{H} & -0.00004 \odot & -1.175370 & 1.229250 \\ \mathrm{H} & 0.000033 & 1.175436 & 1.229264\end{array}$

Optimized geometries of glycinamide conformers and the rotational transition states at B3L YP/6-311+G** level:

Figure 2:

Glycinamide IV

$\begin{array}{lr}\mathrm{N} & -0.934318 \\ \mathrm{C} & 0.025267 \\ \mathrm{H} & 0.836271 \\ \mathrm{H} & -0.382026 \\ \mathrm{H} & -1.883831 \\ \mathrm{H} & -0.964434 \\ \mathrm{C} & 0.697671 \\ \mathrm{O} & 1.389720 \\ \mathrm{~N} & 0.462159 \\ \mathrm{H} & -0.131156 \\ \mathrm{H} & 0.884676\end{array}$

-0.075012
0.783375
1.049501
1.743168
0.080343
0.108096
0.116546
0.752821
-1.205280
-1.586992
-1.766566

1.022760

$\odot .351733$

1.051903

$-0.019914$

$\odot .663165$

2.031946

$-0.831387$

$-1.614186$

$-0.889835$

$-0.135802$

$-1.630383$

Glycinamide $V$

$\begin{array}{rrrr}\mathrm{N} & -.266764 & 1.220848 & -1.480688 \\ \mathrm{C} & -.384335 & .974137 & -.053079 \\ \mathrm{H} & -1.428559 & 1.100292 & .245546 \\ \mathrm{H} & .170924 & 1.744647 & .488675 \\ \mathrm{H} & -.845746 & .624802 & -2.057374 \\ \mathrm{H} & .684085 & 1.240231 & -1.825031 \\ \mathrm{C} & .064408 & -.367531 & .550566 \\ \mathrm{O} & -.035694 & -.569552 & 1.748137 \\ \mathrm{~N} & .568127 & -1.292172 & -.315143 \\ \mathrm{H} & .644576 & -1.112384 & -1.301656 \\ \mathrm{H} & .870294 & -2.181545 & .050639\end{array}$


Glycinamide VI

$\begin{array}{lr}\mathrm{N} & 1.268864 \\ \mathrm{C} & -0.008408 \\ \mathrm{H} & -0.325794 \\ \mathrm{H} & -0.771708 \\ \mathrm{H} & 1.981428 \\ \mathrm{H} & 1.512192 \\ \mathrm{C} & -0.048586 \\ \mathrm{O} & 0.918370 \\ \mathrm{~N} & -1.220007 \\ \mathrm{H} & -2.010963 \\ \mathrm{H} & -1.295388\end{array}$

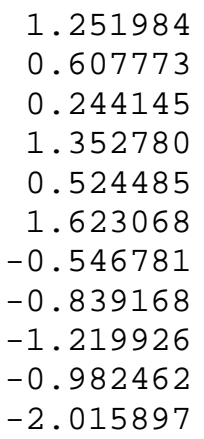

$-0.607428$

$-0.507558$

$-1.504782$

$-0.210430$

$-0.765315$

0.322432

0.474262

1.162377

0.533009

$-0.067719$

1.171153

Glycinamide VII

$\begin{array}{lr}\mathrm{N} & -.066151 \\ \mathrm{C} & -.647960 \\ \mathrm{H} & -.623886 \\ \mathrm{H} & -1.697545 \\ \mathrm{H} & .276694 \\ \mathrm{H} & -.747085 \\ \mathrm{C} & .122773 \\ \mathrm{O} & .669561 \\ \mathrm{~N} & .100481 \\ \mathrm{H} & -.241607 \\ \mathrm{H} & .587745\end{array}$

\section{Figure S1:}

Glycinamide VIII

$\begin{array}{lr}\mathrm{N} & 0.006442995 \\ \mathrm{C} & -0.862684613 \\ \mathrm{H} & -1.477720316 \\ \mathrm{H} & -1.572068236 \\ \mathrm{H} & 0.365808842 \\ \mathrm{H} & 0.425517787 \\ \mathrm{C} & -0.237365889 \\ \mathrm{O} & -0.943055539 \\ \mathrm{~N} & 1.120163461 \\ \mathrm{H} & 1.658064208 \\ \mathrm{H} & 1.587380237\end{array}$

$-0.006276992$

$-0.010257990$

$-0.915306556$

0.820464596

0.853949594

$-0.854910579$

0.073137977

๑. 088096964

$\odot .128353945$

0.097117950

$\odot .163750912$
$-1.881447$

$-.716936$

$-.840353$

$-.521181$

$-1.610330$

$-2.622109$

.539269

.635452

1.557837

1.430478

2. 411153
$-1.499880291$

$-0.353038848$

$-0.356719856$

$-0.417531819$

$-1.871951096$

$-1.833973121$

1. 050976532

2. 044970014

1. 089294503

0.238783871

1. 981154072

Glycinamide IX

$\begin{array}{lr}\mathrm{N} & -\Theta .248752878 \\ \mathrm{C} & -\Theta .766734662 \\ \mathrm{H} & -1.805157158 \\ \mathrm{H} & -\Theta .768393632 \\ \mathrm{H} & 0.544944728 \\ \mathrm{H} & 0.057815974 \\ \mathrm{C} & 0.062703982 \\ \mathrm{O} & 0.665580680 \\ \mathrm{~N} & 0.112844931 \\ \mathrm{H} & -\odot .515531748 \\ \mathrm{H} & 0.607092704\end{array}$

1.140764480
0.673119706
0.335450857
1.476148290
0.591425731
2.104265990
-0.463639804
-0.371433834
-1.591165229
-1.730380161
-2.394755852
$-1.022810504$
0.279916853
0.161001943
1.016998500
$-1.333021364$
$-0.968374542$
0.873177611
1.925973082
$\odot .092563958$
$-0.682774699$
0.449779812 
Glycinamide $\mathrm{X}$

$\begin{array}{rr}\mathrm{N} & 0.104597972 \\ \mathrm{C} & -\odot .876673566 \\ \mathrm{H} & -1.248285415 \\ \mathrm{H} & -1.740737161 \\ \mathrm{H} & \odot .443573783 \\ \mathrm{H} & -\odot .322331871 \\ \mathrm{C} & -\odot .177469932 \\ \mathrm{O} & -0.054350976 \\ \mathrm{~N} & 0.295183874 \\ \mathrm{H} & \odot .376902826 \\ \mathrm{H} & 0.892492571\end{array}$

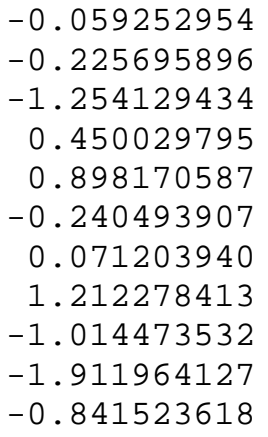

$-1.365824358$

$-0.285413890$

$-0.313985850$

$-0.347044860$

$-1.379111357$

$-2.268659958$

1. 040977521

1.450674344

1. 721885177

1. 271838395

2. 517077814

Glycinamide XI

$\begin{array}{rr}\mathrm{N} & -0.175848004 \\ \mathrm{C} & -\odot .614699729 \\ \mathrm{H} & -0.469737458 \\ \mathrm{H} & -1.683922495 \\ \mathrm{H} & \odot .457050387 \\ \mathrm{H} & -\odot .952119071 \\ \mathrm{C} & \odot .152783243 \\ \mathrm{O} & 0.836446483 \\ \mathrm{~N} & -\odot .002510786 \\ \mathrm{H} & -\odot .500453325 \\ \mathrm{H} & 0.488548589\end{array}$

0.360886913

$-0.403181015$

$-1.479135706$

$-0.266479196$

1.095887837

$\odot .799268623$

0.003331593

1. 010325948

$-0.831081474$

$-1.703580363$

$-\odot .619271183$
$-1.882828858$

$-0.723631502$

$-0.892048380$

$-0.510590504$

$-1.578305593$

$-2.361808763$

0.539475525

0.587892998

1.607468907

1.544908333

2. 461835599

\section{Figure 4:}

Sodium Chloride Clusters:

Na9C19 (100) Cluster

$\begin{array}{lrrr}\mathrm{Na} & 1.513022478 & -4.900804766 & -5.663794325 \\ \mathrm{Na} & 1.513022478 & 0.532157451 & -0.230832108 \\ \mathrm{Na} & 6.945984695 & -4.900804766 & -0.2308321082 \\ \mathrm{Na} & 6.945984695 & 0.532157451 & -5.663794325 \\ \mathrm{Na} & 1.513022478 & -4.900804766 & 5.202130108 \\ \mathrm{Na} & 1.513022478 & 5.965119668 & -5.663794325 \\ \mathrm{Na} & 1.513022478 & 5.9651196682 & 5.2021301089 \\ \mathrm{Na} & 6.945984695 & 5.965119668 & -0.230832108 \\ \mathrm{Na} & 6.945984695 & 0.532157451 & 5.202130108 \\ \mathrm{Cl} & 6.945984695 & 0.532157451 & -0.230832108 \\ \mathrm{Cl} & 6.945984695 & 5.965119668 & 5.202130108 \\ \mathrm{Cl} & 1.513022478 & -4.900804766 & -0.230832108 \\ \mathrm{Cl} & 1.513022478 & 0.532157451 & -5.663794325 \\ \mathrm{Cl} & 6.945984695 & -4.900804766 & -5.663794325 \\ \mathrm{Cl} & 6.945984695 & 5.965119668 & -5.663794325 \\ \mathrm{Cl} & 6.945984695 & -4.900804766 & 5.202130108 \\ \mathrm{Cl} & 1.513022478 & 0.532157451 & 5.202130108 \\ \mathrm{Cl} & 1.513022478 & 5.965119668 & -0.230832108\end{array}$

Na9C19 (111) Cluster

$\mathrm{Na}$

$$
-10.516359784
$$

6.698871906

$-7.672713633$ 


$\begin{array}{lrrr}\mathrm{Na} & 0.349564650 & -4.167052527 & -7.672713633 \\ \mathrm{Na} & 0.349564650 & 6.698871906 & 3.193210800 \\ \mathrm{Na} & 11.215489084 & -4.167052527 & 3.19321080 \odot \\ \mathrm{Na} & 0.349564650 & 1.265909689 & -2.239751416 \\ \mathrm{Na} & -5.083397566 & 6.698871906 & -2.239751416 \\ \mathrm{Na} & 5.782526867 & -4.167052527 & -2.239751416 \\ \mathrm{Na} & -5.083397566 & 1.265909689 & -7.672713633 \\ \mathrm{Na} & 5.782526867 & 1.265909689 & 3.193210800 \\ \mathrm{Cl} & 5.782526867 & 1.265909689 & -2.239751416 \\ \mathrm{Cl} & 5.782526867 & 6.698871906 & 3.193210800 \\ \mathrm{Cl} & 16.648451301 & -4.167052527 & 3.193210800 \\ \mathrm{Cl} & 11.215489084 & 1.265909689 & 3.193210800 \\ \mathrm{Cl} & 0.349564650 & 6.698871906 & -2.239751416 \\ \mathrm{Cl} & 11.215489084 & -4.167052527 & -2.239751416 \\ \mathrm{Cl} & -5.083397566 & 6.698871906 & -7.672713633 \\ \mathrm{Cl} & 5.782526867 & -4.167052527 & -7.672713633 \\ \mathrm{Cl} & 0.349564650 & 1.265909689 & -7.672713633\end{array}$

Adsorbed geometries of urea and glycinamide conformers on the surfaces of sodium chloride at LDA/PWC/DND (DFT) level:

\section{Figure 5:}

Na9C19 (100) Cluster+ C2 (II) complex

$\begin{array}{lrrr}\mathrm{Na} & 0.133567 & -2.798135 & -2.659285 \\ \mathrm{Na} & 0.133567 & 0.076865 & 0.215715 \\ \mathrm{Na} & 3.008567 & -2.798135 & 0.215715 \\ \mathrm{Na} & 3.008567 & 0.076865 & -2.659285 \\ \mathrm{Na} & 0.133567 & -2.798135 & 3.090715 \\ \mathrm{Na} & 0.133567 & 2.951865 & -2.659285 \\ \mathrm{Na} & 0.133567 & 2.951865 & 3.090715 \\ \mathrm{Na} & 3.008567 & 2.951865 & 0.215715 \\ \mathrm{Na} & 3.008567 & 0.076865 & 3.090715 \\ \mathrm{Cl} & 3.008567 & 0.076865 & 0.215715 \\ \mathrm{Cl} & 3.008567 & 2.951865 & 3.090715 \\ \mathrm{Cl} & 0.133567 & -2.798135 & 0.215715 \\ \mathrm{Cl} & 0.133567 & 0.076865 & -2.659285 \\ \mathrm{Cl} & 3.008567 & -2.798135 & -2.659285 \\ \mathrm{Cl} & 3.008567 & 2.951865 & -2.659285 \\ \mathrm{Cl} & 3.008567 & -2.798135 & 3.090715 \\ \mathrm{Cl} & 0.133567 & 0.076865 & 3.090715 \\ \mathrm{Cl} & 0.133567 & 2.951865 & 0.215715 \\ \mathrm{C} & -3.108275 & 0.031861 & -0.584730 \\ \mathrm{O} & -2.177854 & 0.075584 & 0.214745 \\ \mathrm{~N} & -4.408379 & 0.211977 & -0.180016 \\ \mathrm{H} & -5.158511 & -0.245625 & -0.705835 \\ \mathrm{H} & -4.518567 & 0.207102 & 0.838569 \\ \mathrm{~N} & -2.938356 & -0.197407 & -1.919665 \\ \mathrm{H} & -1.951065 & -0.110155 & -2.246654 \\ \mathrm{H} & -3.659375 & 0.137718 & -2.565833\end{array}$

Na9C19 (100) Cluster+ C2 (II) complex in water

$\begin{array}{rrrr}\mathrm{Na} & 0.119088 & -2.854785 & -2.533169 \\ \mathrm{Na} & 0.119088 & 0.020215 & 0.341831 \\ \mathrm{Na} & 2.994088 & -2.854785 & 0.341831\end{array}$




$\begin{array}{lrrr}\mathrm{Na} & 2.994088 & 0.020215 & -2.533169 \\ \mathrm{Na} & 0.119088 & -2.854785 & 3.216831 \\ \mathrm{Na} & 0.119088 & 2.895215 & -2.533169 \\ \mathrm{Na} & 0.119088 & 2.895215 & 3.216831 \\ \mathrm{Na} & 2.994088 & 2.895215 & 0.341831 \\ \mathrm{Na} & 2.994088 & 0.020215 & 3.216831 \\ \mathrm{Cl} & 2.994088 & 0.020215 & 0.341831 \\ \mathrm{Cl} & 2.994088 & 2.895215 & 3.216831 \\ \mathrm{Cl} & 0.119088 & -2.854785 & 0.341831 \\ \mathrm{Cl} & 0.119088 & 0.020215 & -2.533169 \\ \mathrm{Cl} & 2.994088 & -2.854785 & -2.533169 \\ \mathrm{Cl} & 2.994088 & 2.895215 & -2.533169 \\ \mathrm{Cl} & 2.994088 & -2.854785 & 3.216831 \\ \mathrm{Cl} & 0.119088 & 0.020215 & 3.216831 \\ \mathrm{Cl} & 0.119088 & 2.895215 & 0.341831 \\ \mathrm{C} & -3.141493 & 0.015269 & -0.513488 \\ \mathrm{O} & -2.189570 & 0.037332 & 0.280927 \\ \mathrm{~N} & -4.427834 & 0.183653 & -0.088909 \\ \mathrm{H} & -5.201401 & -0.134195 & -0.684318 \\ \mathrm{H} & -4.579995 & 0.055822 & 0.917622 \\ \mathrm{~N} & -2.974799 & -0.169223 & -1.845621 \\ \mathrm{H} & -1.997598 & -0.082404 & -2.190028 \\ \mathrm{H} & -3.727035 & 0.074374 & -2.499803 \\ & & & \end{array}$

Na9C19 (111) Cluster+ C2 (II) complex

$\begin{array}{lrrr}\mathrm{Na} & -6.056086 & 5.107626 & 0.058160 \\ \mathrm{Na} & -0.306086 & 1.041762 & -4.007704 \\ \mathrm{Na} & -0.306086 & 1.041762 & 4.124024 \\ \mathrm{Na} & 5.443914 & -3.024102 & 0.058160 \\ \mathrm{Na} & -0.306086 & 1.041762 & 0.058160 \\ \mathrm{Na} & -3.181086 & 3.074694 & 2.091092 \\ \mathrm{Na} & 2.568914 & -0.991170 & -1.974772 \\ \mathrm{Na} & -3.181086 & 3.074694 & -1.974772 \\ \mathrm{Na} & 2.568914 & -0.991170 & 2.091092 \\ \mathrm{Cl} & 2.568914 & 1.041762 & 0.058160 \\ \mathrm{Cl} & 2.568914 & 1.041762 & 4.124024 \\ \mathrm{Cl} & 8.318914 & -3.024102 & 0.058160 \\ \mathrm{Cl} & 5.443914 & -0.991170 & 2.091092 \\ \mathrm{Cl} & -0.306086 & 3.074694 & 2.091092 \\ \mathrm{Cl} & 5.443914 & -\odot .991170 & -1.974772 \\ \mathrm{Cl} & -3.181086 & 5.107626 & 0.05816 \\ \mathrm{Cl} & 2.568914 & 1.041762 & -4.007704 \\ \mathrm{Cl} & -0.306086 & 3.074694 & -1.974772 \\ \mathrm{C} & -2.374704 & -1.519498 & -0.122076 \\ \mathrm{O} & -1.434859 & -0.802541 & -0.491268 \\ \mathrm{~N} & -3.510795 & -1.010268 & 0.418108 \\ \mathrm{H} & -4.169772 & -1.602598 & 0.933194 \\ \mathrm{H} & -3.519894 & 0.004007 & 0.628164 \\ \mathrm{~N} & -2.312885 & -2.872262 & -0.234379 \\ \mathrm{H} & -1.524064 & -3.236635 & -0.775973 \\ \mathrm{H} & -3.153121 & -3.455302 & -0.187537\end{array}$

Na9C19 (111) Cluster+ C2 (II) complex in water
$\mathrm{Na}$
$-5.992100$
4.944587
0.011511
$\mathrm{Na}$
$-\odot .242100$
$\odot .878723$
$-4.054353$ 


$\begin{array}{lrrr}\mathrm{Na} & -0.242100 & 0.878723 & 4.077375 \\ \mathrm{Na} & 5.507900 & -3.187141 & 0.011511 \\ \mathrm{Na} & -0.242100 & 0.878723 & 0.011511 \\ \mathrm{Na} & -3.117100 & 2.911655 & 2.044443 \\ \mathrm{Na} & 2.632900 & -1.154209 & -2.021421 \\ \mathrm{Na} & -3.117100 & 2.911655 & -2.021421 \\ \mathrm{Na} & 2.632900 & -1.154209 & 2.044443 \\ \mathrm{Cl} & 2.632900 & 0.878723 & 0.011511 \\ \mathrm{Cl} & 2.632900 & 0.878723 & 4.077375 \\ \mathrm{Cl} & 8.382900 & -3.187141 & 0.011511 \\ \mathrm{Cl} & 5.507900 & -1.154209 & 2.044443 \\ \mathrm{Cl} & -0.242100 & 2.911655 & 2.044443 \\ \mathrm{Cl} & 5.507900 & -1.154209 & -2.021421 \\ \mathrm{Cl} & -3.117100 & 4.944587 & 0.011511 \\ \mathrm{Cl} & 2.632900 & 0.878723 & -4.054353 \\ \mathrm{Cl} & -0.242100 & 2.911655 & -2.021421 \\ \mathrm{C} & -0.841148 & -1.802471 & -1.948699 \\ \mathrm{O} & -0.297967 & -0.664323 & -1.901366 \\ \mathrm{~N} & -1.650742 & -2.235292 & -0.969407 \\ \mathrm{H} & -2.017950 & -3.193265 & -0.934932 \\ \mathrm{H} & -1.770962 & -1.660947 & -0.131942 \\ \mathrm{~N} & -0.638548 & -2.635378 & -2.982376 \\ \mathrm{H} & -0.068495 & -2.335642 & -3.778264 \\ \mathrm{H} & -1.104474 & -3.547143 & -3.055123\end{array}$

Na9C19 (100) Cluster+ Cs (I) complex

$\begin{array}{lrrr}\mathrm{Na} & 0.119432 & -2.814646 & -2.520517 \\ \mathrm{Na} & 0.119432 & 0.060354 & 0.354483 \\ \mathrm{Na} & 2.994432 & -2.814646 & -.354483 \\ \mathrm{Na} & 2.994432 & 0.060354 & -2.520517 \\ \mathrm{Na} & 0.119432 & -2.814646 & 3.229483 \\ \mathrm{Na} & 0.119432 & 2.935354 & -2.520517 \\ \mathrm{Na} & 0.119432 & 2.935354 & 3.229483 \\ \mathrm{Na} & 2.994432 & 2.935354 & 0.354483 \\ \mathrm{Na} & 2.994432 & 0.060354 & 3.229483 \\ \mathrm{Cl} & 2.994432 & 0.060354 & 0.354483 \\ \mathrm{Cl} & 2.994432 & 2.935354 & 3.229483 \\ \mathrm{Cl} & 0.119432 & -2.814646 & 0.354483 \\ \mathrm{Cl} & 0.119432 & 0.060354 & -2.520517 \\ \mathrm{Cl} & 2.994432 & -2.814646 & -2.520517 \\ \mathrm{Cl} & 2.994432 & 2.935354 & -2.520517 \\ \mathrm{Cl} & 2.994432 & -2.814646 & 3.229483 \\ \mathrm{Cl} & 0.119432 & 0.060354 & 3.229483 \\ \mathrm{Cl} & 0.119432 & 2.935354 & 0.354483 \\ \mathrm{C} & -3.103864 & 0.100247 & -0.426685 \\ \mathrm{O} & -2.154105 & 0.056881 & 0.352132 \\ \mathrm{~N} & -4.391579 & 0.021106 & 0.032700 \\ \mathrm{H} & -4.475819 & -0.276833 & 1.007751 \\ \mathrm{~N} & -2.949926 & 0.287264 & -1.76539 \\ \mathrm{H} & -1.973936 & 0.200119 & -2.122453 \\ \mathrm{H} & -3.723696 & 0.13310 \odot & -2.416091 \\ \mathrm{H} & -5.175653 & -0.184025 & -0.590290\end{array}$

Na9C19 (100) Cluster+ Cs (I) complex in water 


$\begin{array}{lrrr}\mathrm{Na} & 0.117109 & -2.869807 & -2.537584 \\ \mathrm{Na} & 0.117109 & 0.005193 & 0.337416 \\ \mathrm{Na} & 2.992109 & -2.869807 & 0.337416 \\ \mathrm{Na} & 2.992109 & 0.005193 & -2.537584 \\ \mathrm{Na} & 0.117109 & -2.869807 & 3.212416 \\ \mathrm{Na} & 0.117109 & 2.880193 & -2.537584 \\ \mathrm{Na} & 0.117109 & 2.880193 & 3.212416 \\ \mathrm{Na} & 2.992109 & 2.880193 & 0.337416 \\ \mathrm{Na} & 2.992109 & 0.005193 & 3.212416 \\ \mathrm{Cl} & 2.992109 & 0.005193 & 0.337416 \\ \mathrm{Cl} & 2.992109 & 2.880193 & 3.212416 \\ \mathrm{Cl} & 0.117109 & -2.869807 & 0.337416 \\ \mathrm{Cl} & 0.117109 & 0.005193 & -2.537584 \\ \mathrm{Cl} & 2.992109 & -2.869807 & -2.537584 \\ \mathrm{Cl} & 2.992109 & 2.880193 & -2.537584 \\ \mathrm{Cl} & 2.992109 & -2.869807 & 3.212416 \\ \mathrm{Cl} & 0.117109 & 0.005193 & 3.212416 \\ \mathrm{Cl} & 0.117109 & 2.880193 & 0.337416 \\ \mathrm{C} & -3.125383 & -0.021300 & -0.448201 \\ \mathrm{O} & -2.161713 & -0.039033 & 0.334110 \\ \mathrm{~N} & -4.404866 & -0.120179 & 0.007382 \\ \mathrm{H} & -4.516978 & -0.422878 & 0.980437 \\ \mathrm{~N} & -2.974030 & 0.160038 & -1.780115 \\ \mathrm{H} & -2.003258 & 0.098813 & -2.144564 \\ \mathrm{H} & -3.749814 & -0.002895 & -2.431081 \\ \mathrm{H} & -5.174633 & -0.347014 & -0.631520\end{array}$

Na9C19 (111) Cluster+ Cs (I) complex

$\begin{array}{lrrr}\mathrm{Na} & -5.970897 & 5.021996 & 0.059792 \\ \mathrm{Na} & -0.220897 & 0.956132 & -4.006072 \\ \mathrm{Na} & -0.220897 & 0.956132 & 4.125656 \\ \mathrm{Na} & 5.529103 & -3.109732 & 0.059792 \\ \mathrm{Na} & -0.220897 & 0.956132 & 0.059792 \\ \mathrm{Na} & -3.095897 & 2.989064 & 2.092724 \\ \mathrm{Na} & 2.654103 & -1.076800 & -1.973140 \\ \mathrm{Na} & -3.095897 & 2.989064 & -1.973140 \\ \mathrm{Na} & 2.654103 & -1.076800 & 2.092724 \\ \mathrm{Cl} & 2.654103 & 0.956132 & 0.059792 \\ \mathrm{Cl} & 2.654103 & 0.956132 & 4.125656 \\ \mathrm{Cl} & 8.404103 & -3.109732 & 0.059792 \\ \mathrm{Cl} & 5.529103 & -1.076800 & 2.092724 \\ \mathrm{Cl} & -0.220897 & 2.989064 & 2.092724 \\ \mathrm{Cl} & 5.529103 & -1.076800 & -1.973140 \\ \mathrm{Cl} & -3.095897 & 5.021996 & 0.059792 \\ \mathrm{Cl} & 2.654103 & 0.956132 & -4.006072 \\ \mathrm{Cl} & -0.220897 & 2.989064 & -1.973140 \\ \mathrm{C} & -2.509953 & -1.401333 & -0.231653 \\ \mathrm{O} & -1.601376 & -0.664923 & -0.641123 \\ \mathrm{~N} & -2.805104 & -2.567648 & -0.859948 \\ \mathrm{H} & -2.139559 & -2.877678 & -1.573468 \\ \mathrm{~N} & -3.258796 & -1.094747 & 0.861324 \\ \mathrm{H} & -3.238375 & -0.103514 & 1.166174 \\ \mathrm{H} & -4.130514 & -1.602221 & 1.049533 \\ \mathrm{H} & -3.417811 & -3.274953 & -0.445487\end{array}$

Na9C19 (100) Cluster+ Cs (I) complex in water 


$\begin{array}{lrrr}\mathrm{Na} & -6.008923 & 4.955231 & -0.018731 \\ \mathrm{Na} & -0.258923 & 0.889231 & -4.084731 \\ \mathrm{Na} & -0.258923 & 0.889231 & 4.047269 \\ \mathrm{Na} & 5.491077 & -3.176769 & -0.018731 \\ \mathrm{Na} & -0.258923 & 0.889231 & -0.018731 \\ \mathrm{Na} & -3.133923 & 2.922231 & 2.014269 \\ \mathrm{Na} & 2.616077 & -1.143769 & -2.051731 \\ \mathrm{Na} & -3.133923 & 2.922231 & -2.051731 \\ \mathrm{Na} & 2.616077 & -1.143769 & 2.014269 \\ \mathrm{Cl} & 2.616077 & 0.889231 & -0.018731 \\ \mathrm{Cl} & 2.616077 & 0.889231 & 4.047269 \\ \mathrm{Cl} & 8.366077 & -3.176769 & -0.018731 \\ \mathrm{Cl} & 5.491077 & -1.143769 & 2.014269 \\ \mathrm{Cl} & -0.258923 & 2.922231 & 2.014269 \\ \mathrm{Cl} & 5.491077 & -1.143769 & -2.051731 \\ \mathrm{Cl} & -3.133923 & 4.955231 & -0.018731 \\ \mathrm{Cl} & 2.616077 & 0.889231 & -4.084731 \\ \mathrm{Cl} & -0.258923 & 2.922231 & -2.051731 \\ \mathrm{C} & -1.262324 & -1.571473 & -2.035808 \\ \mathrm{O} & -0.690605 & -0.447005 & -2.030779 \\ \mathrm{~N} & -2.200710 & -1.885377 & -1.125749 \\ \mathrm{H} & -2.754850 & -2.747105 & -1.197385 \\ \mathrm{H} & -2.613876 & -1.134596 & -0.566520 \\ \mathrm{~N} & -0.918414 & -2.530927 & -2.910443 \\ \mathrm{H} & -0.257379 & -2.312947 & -3.661350 \\ \mathrm{H} & -1.455472 & -3.400768 & -3.006515 \\ & & & \end{array}$

Na9C19 (100) Cluster+ C2v (III) complex

$\begin{array}{lrrr}\mathrm{Na} & 0.094799 & -3.282777 & -2.817576 \\ \mathrm{Na} & 0.094799 & -0.407777 & 0.057424 \\ \mathrm{Na} & 2.969799 & -3.282777 & 0.057424 \\ \mathrm{Na} & 2.969799 & -0.407777 & -2.817576 \\ \mathrm{Na} & 0.094799 & -3.282777 & 2.932424 \\ \mathrm{Na} & 0.094799 & 2.467223 & -2.817576 \\ \mathrm{Na} & 0.094799 & 2.467223 & 2.932424 \\ \mathrm{Na} & 2.969799 & 2.467223 & 0.057424 \\ \mathrm{Na} & 2.969799 & -\Theta .407777 & 2.932424 \\ \mathrm{Cl} & 2.969799 & -0.407777 & 0.057424 \\ \mathrm{Cl} & 2.969799 & 2.467223 & 2.932424 \\ \mathrm{Cl} & 0.094799 & -3.282777 & 0.057424 \\ \mathrm{Cl} & 0.094799 & -0.407777 & -2.817576 \\ \mathrm{Cl} & 2.969799 & -3.282777 & -2.817576 \\ \mathrm{Cl} & 2.969799 & 2.467223 & -2.817576 \\ \mathrm{Cl} & 2.969799 & -3.282777 & 2.932424 \\ \mathrm{Cl} & 0.094799 & -0.407777 & 2.932424 \\ \mathrm{Cl} & 0.094799 & 2.467223 & 0.057424 \\ \mathrm{C} & -3.116027 & 0.372964 & 0.054415 \\ \mathrm{O} & -2.165111 & -0.406355 & 0.056698 \\ \mathrm{~N} & -2.965187 & 1.721985 & 0.039050 \\ \mathrm{H} & -1.986538 & 2.083028 & 0.045997 \\ \mathrm{~N} & -4.399037 & -\Theta .092939 & 0.062380 \\ \mathrm{H} & -4.513763 & -1.107642 & 0.070273 \\ \mathrm{H} & -5.227033 & 0.503915 & 0.054549 \\ \mathrm{H} & -3.760823 & 2.361812 & 0.052462\end{array}$


Na9C19 (100) Cluster+ C2v (III) complex in water

$\begin{array}{lrrr}\mathrm{Na} & 0.116770 & -3.209573 & -2.874097 \\ \mathrm{Na} & 0.116770 & -0.334573 & 0.000903 \\ \mathrm{Na} & 2.991770 & -3.209573 & 0.000903 \\ \mathrm{Na} & 2.991770 & -0.334573 & -2.874097 \\ \mathrm{Na} & 0.116770 & -3.209573 & 2.875903 \\ \mathrm{Na} & 0.116770 & 2.540427 & -2.874097 \\ \mathrm{Na} & 0.116770 & 2.540427 & 2.875903 \\ \mathrm{Na} & 2.991770 & 2.540427 & 0.00 \odot 903 \\ \mathrm{Na} & 2.991770 & -0.334573 & 2.875903 \\ \mathrm{Cl} & 2.991770 & -0.334573 & 0.000903 \\ \mathrm{Cl} & 2.991770 & 2.540427 & 2.875903 \\ \mathrm{Cl} & 0.116770 & -3.209573 & 0.000903 \\ \mathrm{Cl} & 0.116770 & -0.334573 & -2.874097 \\ \mathrm{Cl} & 2.991770 & -3.209573 & -2.874097 \\ \mathrm{Cl} & 2.991770 & 2.540427 & -2.874097 \\ \mathrm{Cl} & 2.991770 & -3.209573 & 2.875903 \\ \mathrm{Cl} & 0.116770 & -0.334573 & 2.875903 \\ \mathrm{Cl} & 0.116770 & 2.540427 & 0.000903 \\ \mathrm{C} & -3.116498 & 0.457772 & -0.002503 \\ \mathrm{O} & -2.150904 & -\odot .325605 & 0.000493 \\ \mathrm{~N} & -2.959993 & 1.798806 & -0.020028 \\ \mathrm{H} & -1.989398 & 2.166184 & -0.012942 \\ \mathrm{~N} & -4.393866 & 0.000379 & 0.009094 \\ \mathrm{H} & -4.545768 & -1.012031 & 0.017969 \\ \mathrm{H} & -5.210583 & 0.618863 & 0.001249 \\ \mathrm{H} & -3.753131 & 2.447532 & -0.004673\end{array}$

Na9C19 (111) Cluster+ C2v (III) complex

$\begin{array}{lrrr}\mathrm{Na} & -5.987215 & 5.062517 & -0.008143 \\ \mathrm{Na} & -0.237215 & 0.996653 & -4.074007 \\ \mathrm{Na} & -0.237215 & 0.996653 & 4.057721 \\ \mathrm{Na} & 5.512785 & -3.069211 & -0.008143 \\ \mathrm{Na} & -0.237215 & 0.996653 & -0.008143 \\ \mathrm{Na} & -3.112215 & 3.029585 & 2.024789 \\ \mathrm{Na} & 2.637785 & -1.036279 & -2.041075 \\ \mathrm{Na} & -3.112215 & 3.029585 & -2.041075 \\ \mathrm{Na} & 2.637785 & -1.036279 & 2.024789 \\ \mathrm{Cl} & 2.637785 & 0.996653 & -0.008143 \\ \mathrm{Cl} & 2.637785 & 0.996653 & 4.057721 \\ \mathrm{Cl} & 8.387785 & -3.069211 & -0.008143 \\ \mathrm{Cl} & 5.512785 & -1.036279 & 2.024789 \\ \mathrm{Cl} & -0.237215 & 3.029585 & 2.024789 \\ \mathrm{Cl} & 5.512785 & -1.036279 & -2.041075 \\ \mathrm{Cl} & -3.112215 & 5.062517 & -0.008143 \\ \mathrm{Cl} & 2.637785 & 0.996653 & -4.074007 \\ \mathrm{Cl} & -0.237215 & 3.029585 & -2.041075 \\ \mathrm{C} & -2.201822 & -1.627476 & 0.215067 \\ \mathrm{O} & -1.274424 & -0.891798 & 0.579548 \\ \mathrm{~N} & -3.226617 & -1.175588 & -0.558153 \\ \mathrm{H} & -3.335258 & -0.147724 & -0.620803 \\ \mathrm{~N} & -2.232323 & -2.939046 & 0.553689 \\ \mathrm{H} & -1.413086 & -3.317505 & 1.035694 \\ \mathrm{H} & -2.936559 & -3.596302 & 0.210597 \\ \mathrm{H} & -4.078685 & -1.731737 & -0.683486\end{array}$


Na9C19 (111) Cluster+ C2v (III) complex in water

$\begin{array}{lrrr}\mathrm{Na} & -5.994269 & 4.945346 & 0.016077 \\ \mathrm{Na} & -0.244269 & 0.879346 & -4.049923 \\ \mathrm{Na} & -0.244269 & 0.879346 & 4.081077 \\ \mathrm{Na} & 5.505731 & -3.185654 & 0.016077 \\ \mathrm{Na} & -0.244269 & 0.879346 & 0.016077 \\ \mathrm{Na} & -3.119269 & 2.912346 & 2.049077 \\ \mathrm{Na} & 2.630731 & -1.152654 & -2.016923 \\ \mathrm{Na} & -3.119269 & 2.912346 & -2.016923 \\ \mathrm{Na} & 2.630731 & -1.152654 & 2.049077 \\ \mathrm{Cl} & 2.630731 & 0.879346 & 0.016077 \\ \mathrm{Cl} & 2.630731 & 0.879346 & 4.081077 \\ \mathrm{Cl} & 8.380731 & -3.185654 & 0.016077 \\ \mathrm{Cl} & 5.505731 & -1.152654 & 2.049077 \\ \mathrm{Cl} & -0.244269 & 2.912346 & 2.049077 \\ \mathrm{Cl} & 5.505731 & -1.152654 & -2.016923 \\ \mathrm{Cl} & -3.119269 & 4.945346 & 0.016077 \\ \mathrm{Cl} & 2.630731 & 0.879346 & -4.049923 \\ \mathrm{Cl} & -0.244269 & 2.912346 & -2.016923 \\ \mathrm{C} & -1.020961 & -1.701832 & -2.015368 \\ \mathrm{O} & -0.512796 & -0.547850 & -1.979181 \\ \mathrm{~N} & -1.895110 & -2.121166 & -1.087041 \\ \mathrm{H} & -2.303639 & -3.062392 & -1.099811 \\ \mathrm{H} & -2.273000 & -1.457352 & -0.405164 \\ \mathrm{~N} & -0.690083 & -2.581778 & -2.976540 \\ \mathrm{H} & -0.021121 & -2.314521 & -3.703900 \\ \mathrm{H} & -1.181040 & -3.474991 & -3.098481\end{array}$

Na9C19 (100) Cluster + (IV) complex

$\begin{array}{lrrr}\mathrm{Na} & 0.721676 & -2.863394 & -2.454780 \\ \mathrm{Na} & 0.721676 & 0.011606 & 0.420220 \\ \mathrm{Na} & 3.596676 & -2.863394 & 0.420220 \\ \mathrm{Na} & 3.596676 & 0.011606 & -2.454780 \\ \mathrm{Na} & 0.721676 & -2.863394 & 3.295220 \\ \mathrm{Na} & 0.721676 & 2.886606 & -2.454780 \\ \mathrm{Na} & 0.721676 & 2.886606 & 3.295220 \\ \mathrm{Na} & 3.596676 & 2.886606 & 0.420220 \\ \mathrm{Na} & 3.596676 & 0.011606 & 3.295220 \\ \mathrm{Cl} & 3.596676 & 0.011606 & 0.420220 \\ \mathrm{Cl} & 3.596676 & 2.886606 & 3.295220 \\ \mathrm{Cl} & 0.721676 & -2.863394 & 0.420220 \\ \mathrm{Cl} & 0.721676 & 0.011606 & -2.454780 \\ \mathrm{Cl} & 3.596676 & -2.863394 & -2.454780 \\ \mathrm{Cl} & 3.596676 & 2.886606 & -2.454780 \\ \mathrm{Cl} & 3.596676 & -2.863394 & 3.295220 \\ \mathrm{Cl} & 0.721676 & 0.011606 & 3.295220 \\ \mathrm{Cl} & 0.721676 & 2.886606 & 0.420220 \\ \mathrm{~N} & -4.884504 & 0.088415 & -1.006827 \\ \mathrm{C} & -3.938351 & -0.153175 & 0.064079 \\ \mathrm{H} & -4.061272 & -1.186067 & 0.436736 \\ \mathrm{H} & -4.044196 & 0.505399 & 0.949473 \\ \mathrm{H} & -5.140455 & 1.082557 & -1.064654 \\ \mathrm{H} & -5.752495 & -0.446517 & -0.884154 \\ \mathrm{C} & -2.499011 & -0.049217 & -0.387480\end{array}$




$\begin{array}{rrrr}0 & -1.588291 & -0.039543 & 0.439509 \\ \mathrm{~N} & -2.349064 & 0.004629 & -1.714966 \\ \mathrm{H} & -3.225953 & -0.053292 & -2.259289 \\ \mathrm{H} & -1.389153 & 0.015845 & -2.121661\end{array}$

Na9C19 (100) Cluster + complex(IV)in water

$\begin{array}{lrrr}\mathrm{Na} & 0.721166 & -2.858709 & -2.455267 \\ \mathrm{Na} & 0.721166 & 0.016291 & 0.419733 \\ \mathrm{Na} & 3.596166 & -2.858709 & 0.419733 \\ \mathrm{Na} & 3.596166 & 0.016291 & -2.455267 \\ \mathrm{Na} & 0.721166 & -2.858709 & 3.294733 \\ \mathrm{Na} & 0.721166 & 2.891291 & -2.455267 \\ \mathrm{Na} & 0.721166 & 2.891291 & 3.294733 \\ \mathrm{Na} & 3.596166 & 2.891291 & 0.419733 \\ \mathrm{Na} & 3.596166 & 0.016291 & 3.294733 \\ \mathrm{Cl} & 3.596166 & 0.016291 & 0.419733 \\ \mathrm{Cl} & 3.596166 & 2.891291 & 3.294733 \\ \mathrm{Cl} & 0.721166 & -2.858709 & 0.419733 \\ \mathrm{Cl} & 0.721166 & 0.016291 & -2.455267 \\ \mathrm{Cl} & 3.596166 & -2.858709 & -2.455267 \\ \mathrm{Cl} & 3.596166 & 2.891291 & -2.455267 \\ \mathrm{Cl} & 3.596166 & -2.858709 & 3.294733 \\ \mathrm{Cl} & 0.721166 & 0.016291 & 3.294733 \\ \mathrm{Cl} & 0.721166 & 2.891291 & 0.419733 \\ \mathrm{~N} & -4.922768 & 0.082148 & -0.923883 \\ \mathrm{C} & -3.946623 & -0.149268 & 0.116833 \\ \mathrm{H} & -4.063864 & -1.178116 & 0.504986 \\ \mathrm{H} & -4.031650 & 0.522746 & 0.995492 \\ \mathrm{H} & -5.178126 & 1.078071 & -0.978517 \\ \mathrm{H} & -5.790163 & -0.440378 & -0.744067 \\ \mathrm{C} & -2.522367 & -0.052017 & -0.369615 \\ \mathrm{O} & -1.584860 & -0.037750 & 0.440203 \\ \mathrm{~N} & -2.388512 & -0.002364 & -1.692647 \\ \mathrm{H} & -3.269376 & -\odot .042916 & -2.235700 \\ \mathrm{H} & -1.439432 & 0.013456 & -2.117403\end{array}$

Na9C19 (111) Cluster + complex (IV)

$\begin{array}{rrrr}\mathrm{Na} & -5.652848 & 3.892707 & -3.919542 \\ \mathrm{Na} & 0.094846 & -1.855050 & -3.912406 \\ \mathrm{Na} & 0.097152 & 3.892707 & 1.830458 \\ \mathrm{Na} & 5.847152 & -1.857293 & 1.830458 \\ \mathrm{Na} & 0.097152 & 1.017707 & -1.044542 \\ \mathrm{Na} & -2.777848 & 3.892707 & -1.044542 \\ \mathrm{Na} & 2.972152 & -1.857293 & -1.044542 \\ \mathrm{Na} & -2.777848 & 1.017707 & -3.919542 \\ \mathrm{Na} & 2.972152 & 1.017707 & 1.830458 \\ \mathrm{Cl} & 2.972152 & 1.017707 & -1.044542 \\ \mathrm{Cl} & 2.972152 & 3.892707 & 1.830458 \\ \mathrm{Cl} & 8.722152 & -1.857293 & 1.830458 \\ \mathrm{Cl} & 5.847152 & 1.017707 & 1.830458 \\ \mathrm{Cl} & 0.097152 & 3.892707 & -1.044542 \\ \mathrm{Cl} & 5.847152 & -1.857293 & -1.044542 \\ \mathrm{Cl} & -2.777848 & 3.892707 & -3.919542 \\ \mathrm{Cl} & 2.972152 & -1.857293 & -3.919542 \\ \mathrm{Cl} & 0.097152 & 1.017707 & -3.919542\end{array}$




$\begin{array}{lrrr}\mathrm{N} & -3.415372 & -1.980613 & 2.700662 \\ \mathrm{C} & -2.575768 & -0.933766 & 2.150188 \\ \mathrm{H} & -1.798347 & -0.659257 & 2.883724 \\ \mathrm{H} & -3.099826 & 0.006524 & 1.896851 \\ \mathrm{H} & -4.378267 & -1.895007 & 2.357803 \\ \mathrm{H} & -3.456876 & -1.924581 & 3.723090 \\ \mathrm{C} & -1.839412 & -1.363950 & 0.899904 \\ \mathrm{O} & -1.236057 & -0.560853 & 0.205276 \\ \mathrm{~N} & -1.919240 & -2.687027 & 0.689405 \\ \mathrm{H} & -2.458118 & -3.212978 & 1.394954 \\ \mathrm{H} & -1.444151 & -3.109464 & -0.107241\end{array}$

$\begin{array}{crrr}\mathrm{Na9Cl9}(111) & \text { Cluster }+(\text { IV }) & \text { complex } & \text { in water } \\ \mathrm{Na} & -5.652848 & 3.892707 & -3.919542 \\ \mathrm{Na} & 0.094846 & -1.855050 & -3.912406 \\ \mathrm{Na} & 0.097152 & 3.892707 & 1.830458 \\ \mathrm{Na} & 5.847152 & -1.857293 & 1.830458 \\ \mathrm{Na} & 0.097152 & 1.017707 & -1.044542 \\ \mathrm{Na} & -2.777848 & 3.892707 & -1.044542 \\ \mathrm{Na} & 2.972152 & -1.857293 & -1.044542 \\ \mathrm{Na} & -2.777848 & 1.017707 & -3.919542 \\ \mathrm{Na} & 2.972152 & 1.017707 & 1.830458 \\ \mathrm{Cl} & 2.972152 & 1.017707 & -1.044542 \\ \mathrm{Cl} & 2.972152 & 3.892707 & 1.830458 \\ \mathrm{Cl} & 8.722152 & -1.857293 & 1.830458 \\ \mathrm{Cl} & 5.847152 & 1.017707 & 1.830458 \\ \mathrm{Cl} & 0.097152 & 3.892707 & -1.044542 \\ \mathrm{Cl} & 5.847152 & -1.857293 & -1.044542 \\ \mathrm{Cl} & -2.777848 & 3.892707 & -3.919542 \\ \mathrm{Cl} & 2.972152 & -1.857293 & -3.919542 \\ \mathrm{Cl} & 0.097152 & 1.017707 & -3.919542 \\ \mathrm{~N} & -3.415372 & -1.980613 & 2.700662 \\ \mathrm{C} & -2.575768 & -0.933766 & 2.150188 \\ \mathrm{H} & -1.798347 & -0.659257 & 2.883724 \\ \mathrm{H} & -3.099826 & 0.006524 & 1.896851 \\ \mathrm{H} & -4.378267 & -1.895007 & 2.357803 \\ \mathrm{H} & -3.456876 & -1.924581 & 3.723090 \\ \mathrm{C} & -1.839412 & -1.363950 & 0.899904 \\ \mathrm{O} & -1.236057 & -0.560853 & 0.205276 \\ \mathrm{~N} & -1.919240 & -2.687027 & 0.689405 \\ \mathrm{H} & -2.458118 & -3.212978 & 1.394954 \\ \mathrm{H} & -1.444151 & -3.109464 & -0.107241 \\ & & & \end{array}$

Na9C19 (100) Cluster + (VI) complex

$\begin{array}{rrrr}\mathrm{Na} & 0.536812 & -2.794954 & -2.708266 \\ \mathrm{Na} & 0.536812 & 0.080046 & 0.166734 \\ \mathrm{Na} & 3.411812 & -2.794954 & 0.166734 \\ \mathrm{Na} & 3.411812 & 0.080046 & -2.708266 \\ \mathrm{Na} & 0.536812 & -2.794954 & 3.041734 \\ \mathrm{Na} & 0.536812 & 2.955046 & -2.708266 \\ \mathrm{Na} & 0.536812 & 2.955046 & 3.041734 \\ \mathrm{Na} & 3.411812 & 2.955046 & 0.166734 \\ \mathrm{Na} & 3.411812 & 0.080046 & 3.041734 \\ \mathrm{Cl} & 3.411812 & 0.080046 & 0.166734 \\ \mathrm{Cl} & 3.411812 & 2.955046 & 3.041734 \\ \mathrm{Cl} & 0.536812 & -2.794954 & 0.166734\end{array}$




$\begin{array}{lrrr}\mathrm{Cl} & 0.536812 & 0.080046 & -2.708266 \\ \mathrm{Cl} & 3.411812 & -2.794954 & -2.708266 \\ \mathrm{Cl} & 3.411812 & 2.955046 & -2.708266 \\ \mathrm{Cl} & 3.411812 & -2.794954 & 3.041734 \\ \mathrm{Cl} & 0.536812 & 0.080046 & 3.041734 \\ \mathrm{Cl} & 0.536812 & 2.955046 & 0.166734 \\ \mathrm{~N} & -4.221139 & 1.241867 & -0.141200 \\ \mathrm{C} & -4.111307 & -0.182124 & -0.002693 \\ \mathrm{H} & -4.654604 & -0.679482 & -0.829569 \\ \mathrm{H} & -4.625848 & -0.499456 & 0.926964 \\ \mathrm{H} & -3.781118 & 1.523912 & -1.029573 \\ \mathrm{H} & -3.627631 & 1.679505 & 0.581143 \\ \mathrm{C} & -2.686673 & -0.695794 & 0.054511 \\ \mathrm{O} & -1.750246 & 0.092696 & 0.160898 \\ \mathrm{~N} & -2.520509 & -2.029109 & -0.002106 \\ \mathrm{H} & -3.326717 & -2.657116 & -0.068731 \\ \mathrm{H} & -1.551660 & -2.416419 & 0.074674\end{array}$

Na9C19 (100) Cluster + (VI) complex in water

$\begin{array}{lrrr}\mathrm{Na} & 0.582430 & -2.685519 & -2.802198 \\ \mathrm{Na} & 0.582430 & 0.189481 & 0.072802 \\ \mathrm{Na} & 3.457430 & -2.685519 & 0.072802 \\ \mathrm{Na} & 3.457430 & 0.189481 & -2.802198 \\ \mathrm{Na} & 0.582430 & -2.685519 & 2.947802 \\ \mathrm{Na} & 0.582430 & 3.064481 & -2.802198 \\ \mathrm{Na} & 0.582430 & 3.064481 & 2.947802 \\ \mathrm{Na} & 3.457430 & 3.064481 & 0.072802 \\ \mathrm{Na} & 3.457430 & 0.189481 & 2.947802 \\ \mathrm{Cl} & 3.457430 & 0.189481 & 0.072802 \\ \mathrm{Cl} & 3.457430 & 3.064481 & 2.947802 \\ \mathrm{Cl} & 0.582430 & -2.685519 & 0.072802 \\ \mathrm{Cl} & 0.582430 & 0.189481 & -2.802198 \\ \mathrm{Cl} & 3.457430 & -2.685519 & -2.802198 \\ \mathrm{Cl} & 3.457430 & 3.064481 & -2.802198 \\ \mathrm{Cl} & 3.457430 & -2.685519 & 2.947802 \\ \mathrm{Cl} & 0.582430 & 0.189481 & 2.947802 \\ \mathrm{Cl} & 0.582430 & 3.064481 & 0.072802 \\ \mathrm{~N} & -2.531162 & 1.308325 & -2.524036 \\ \mathrm{C} & -3.038962 & -0.025038 & -2.282360 \\ \mathrm{H} & -3.086542 & -0.586981 & -3.232591 \\ \mathrm{H} & -4.076992 & 0.027894 & -1.898147 \\ \mathrm{H} & -1.548021 & 1.167511 & -2.828660 \\ \mathrm{H} & -2.444412 & 1.765769 & -1.599850 \\ \mathrm{C} & -2.212927 & -0.771479 & -1.266932 \\ \mathrm{O} & -1.663229 & -0.159347 & -0.342896 \\ \mathrm{~N} & -2.107272 & -2.098990 & -1.416108 \\ \mathrm{H} & -2.678113 & -2.607398 & -2.099663 \\ \mathrm{H} & -1.545256 & -2.619646 & -0.714779\end{array}$

Na9C19 (111) Cluster + (VI) complex

$\begin{array}{rrrr}\mathrm{Na} & -5.953274 & 5.428118 & 0.124734 \\ \mathrm{Na} & -0.203274 & 1.362254 & -3.941130 \\ \mathrm{Na} & -0.203274 & 1.362254 & 4.190598 \\ \mathrm{Na} & 5.546726 & -2.703610 & 0.124734 \\ \mathrm{Na} & -0.203274 & 1.362254 & 0.124734\end{array}$




$\begin{array}{lrrr}\mathrm{Na} & -3.078274 & 3.395186 & 2.157666 \\ \mathrm{Na} & 2.671726 & -0.670678 & -1.908198 \\ \mathrm{Na} & -3.078274 & 3.395186 & -1.908198 \\ \mathrm{Na} & 2.671726 & -0.670678 & 2.157666 \\ \mathrm{Cl} & 2.671726 & 1.362254 & 0.124734 \\ \mathrm{Cl} & 2.671726 & 1.362254 & 4.190598 \\ \mathrm{Cl} & 8.421726 & -2.703610 & 0.124734 \\ \mathrm{Cl} & 5.546726 & -0.670678 & 2.157666 \\ \mathrm{Cl} & -0.203274 & 3.395186 & 2.157666 \\ \mathrm{Cl} & 5.546726 & -0.670678 & -1.908198 \\ \mathrm{Cl} & -3.078274 & 5.428118 & 0.124734 \\ \mathrm{Cl} & 2.671726 & 1.362254 & -3.941130 \\ \mathrm{Cl} & -0.203274 & 3.395186 & -1.908198 \\ \mathrm{~N} & -1.897674 & -2.928400 & -0.313436 \\ \mathrm{C} & -3.093881 & -2.152142 & -0.413488 \\ \mathrm{H} & -3.873898 & -2.593915 & 0.235584 \\ \mathrm{H} & -3.495414 & -2.231298 & -1.444705 \\ \mathrm{H} & -1.565553 & -2.960052 & 0.659990 \\ \mathrm{H} & -1.147671 & -2.488213 & -0.864623 \\ \mathrm{C} & -2.943480 & -0.676672 & -0.096506 \\ \mathrm{O} & -1.825276 & -0.165450 & 0.040552 \\ \mathrm{~N} & -4.085973 & 0.025401 & -0.012796 \\ \mathrm{H} & -4.995476 & -0.436542 & -0.128586 \\ \mathrm{H} & -4.070730 & 1.057549 & 0.121368\end{array}$

